\title{
Microbiological Study of Diabetic Foot Ulcer
}

\author{
Mohammuddunnobi ${ }^{\mathrm{a}}$, Jahan $\mathrm{T}^{\mathrm{b}}$, Al-Amin $\mathrm{A}^{\mathrm{c}}$
}

\begin{abstract}
Background: Diabetic foot is one of the most feared complications of diabetes and is the leading cause of hospitalization in diabetic patients. Limb-threatening infection in diabetic patients are usually polymicrobial involving both multiple aerobic and anaerobic organisms.
\end{abstract}

Methods: The present study was a cross sectional study, conducted in the Department of Surgery and Microbiology at BIRDEM General Hospital, Dhaka, over a period of 9 months during January 2017-September' 2017. The study included a total of 77 adult patients of clinically diagnosed diabetic foot patients presenting to outpatient department and emergency ward. The standard case definition of diabetic foot is 'any pathology occurring in the foot of a patient suffering from diabetes mellitus or as a result of long term complication of diabetes mellitus'.

Results: 17(22.1\%) patients had Klebsiella pneumonia, 14(18.2\%) had Pseudomonas aeruginosa, 11(14.3\%) had Staphylococcus aureus, 10(13.0\%) had Escherichia coli, 6(7.8\%) had Coagulase-negative staphylococci and $8(10.4 \%)$ had Providencia spp. In Escherichia coli 100\% sensitivity to imipenem, $70 \%$ to amoxicillinclavulanic acid, amikacin, piperacillin-tazobactam. In Coagulase-negative Staphylococci 83.3\% sensitivity to tetracycline, $66.7 \%$ to ceftriaxone. In Proteus mirabilis $100 \%$ sensitivity to tetracycline, amikacin, ceftriaxone, imipenem, piperacillin-tazobactam. In Enterococcus spp.75.0\% sensitivity to tetracycline. In Citrobacter spp. 100\% sensitivity to imipenem.

Conclusion: Common organism found in diabetic foot ulcer patients were Klebsiella pneumonia, Pseudomonas aeruginosa, Staphylococcus aureus, Escherichia coli, Coagulase-negative staphylococci and Providencia spp. In tetracycline, amikacin, ceftriaxone, imipenem, piperacillin-tazobactam was $100 \%$ sensitive in Proteus mirabilis and only imipenem found in Citrobacter spp.

Keywords: Diabetic foot ulcer, Organism.

(BIRDEM Med J 2018; 8(3): 251-156)

\section{Introduction}

Diabetic foot is one of the most feared complications of diabetes and is the leading cause of hospitalization in diabetic patients. ${ }^{1}$ Diabetic Foot Syndrome (DFS) is a complex and heterogeneous disorder that affects $15 \%$ of patients with diabetes during their lifetime. ${ }^{2}$ Wounds

\section{Author Information}

a. Dr. Mohammuddunnobi, Resident Surgeon (Casualty), BIRDEM General Hospital.

b. Dr. Tasnuva Jahan, Medical officer, DMCH.

c. Prof. Abdullah -Al-Amin, Prof. of Surgery Dept, BIRDEM General Hospital, Dhaka.

Address of Correspondence: Mohammuddunnobi, Resident surgeon (casualty). Room No. 190. Surgical Emergency, BIRDEM General Hospital, Dhaka, Bangladesh. E-mail: drskfiroz2005@ yahoo.com.

Received: June 28, 2018

Accepted: July 31, 2018 of diabetic foot very often get infected due to several factors including high blood sugar level, suppressed immunity, inadequate blood supply and neuropathy. ${ }^{3}$ Polymicrobial infections involving both aerobic and anaerobic bacteria are very common in diabetic foot ulcers, in many centres of developing countries, anaerobes are rarely isolated due to technical difficulties. ${ }^{4}$ Approximately $85 \%$ of all diabetes-related lower-extremity amputations are preceded by foot ulcers. Diabetic foot ulcers are at high risk of infection secondary to high glucose levels and poor tissue perfusion. ${ }^{5}$ Limb-threatening diabetic infections are usually polymicrobial involving multiple aerobic and anaerobic organisms. Staphylococcus aureus, Streptococcus spp., Enterobacteriaceae spp., Bacteroides fragilis, Peptococcus spp. and Peptostreptococcus spp. are the common organisms 
cultured from diabetic ulcers. ${ }^{6}$ The aim of the study was undertaken to identify the aerobic, anaerobic, and fungal pathogens involved in the different grades of diabetic foot ulcers and to find out the antimicrobial sensitivity pattern of the bacterial isolates.

\section{Methods}

The present study was a cross sectional study, conducted in the Department of Surgery and Microbiology at BIRDEM General Hospital, Dhaka, over a period of 9 months during January 2017- September 2017. The study included a total of 77 adult patients of clinically diagnosed diabetic foot patients presenting to outpatient department and emergency ward. The standard case definition of diabetic foot is 'any pathology occurring in the foot of a patient suffering from diabetes mellitus or as a result of long term complication of diabetes mellitus'. Samples were taken from enrolled subjects using the following criteria: patient with diabetic foot pathology. All patients signed an informed consent. Samples were inoculated immediately at the bedside, on pre-reduced Brucella blood agar (Hi-Media) plates enriched with $5 \mathrm{ig} / \mathrm{ml}$ hemin and $1 \mathrm{ig} / \mathrm{ml}$ menadione. Each plate was immediately put inside the modified candle jar, and before closing the jar lid, anaerobiosis was initiated by lighting a small white wax candle and putting $5 \mathrm{~g}$ of acidified copper-coated steel wool on an open plate kept inside. This simple inhouse developed method was standardized earlier and was found suitable for the initiation of anaerobiosis at bedside. Simultaneously, a separate inoculated plate was placed in a jar with GasPak system (Anaerogas Pack- HiMedia) and another inoculated plate for aerobic incubation. After $48 \mathrm{~h}$ of incubation at $37^{\circ} \mathrm{C}$, the anaerobic plates were examined for growth and used for aero-tolerance study by aerobic incubation on blood agar plate after subculture. Colony morphology was noted and bacterial morphology was observed from Gram-stained smears. Aerobic bacteria were identified based on the results of standard biochemical tests. The sensitivity tests were performed by modified KirbyBauer disk diffusion method following the Clinical and Laboratory Standards Institute guideline. Suspected anaerobic isolates, verified by aero-tolerance study, were put into a fresh set of modified candle jars to perform biochemical tests. The biochemical tests included fermentation, indole, nitrate disk reduction, catalase and urease tests. Special-potency disk test (vancomycin, 5 ìg; kanamycin, 1000 ìg; and colistin, 10 ìg), sodium polyanethol sulphonate disk test, bile esculin hydrolysis test, lipase and lecithinase test, pigment production test and colony observation of fluorescence study were also included for presumptive identification of anaerobes up to the genus level8. Isolated anaerobes were tested for antibiotic susceptibility by the $E$-test (BioMérieux, France) in the same modified candle jar system. Antibiotics tested were metronidazole, clindamycin, cefoxitin, imipenem and penicillin.

\section{Results}

Majority 27(35.1\%) patients belonged to age 51-60 years with mean age was found $51.57 \pm 12.13$ years. Males were predominant $(70.1 \%)$, male: female ratio was 2.3:1. Almost two third (64.9\%) patients had trauma, 23(29.9\%) were smoker and $11(14.3 \%)$ had family history of diabetes (Table I). More than two third (68.6\%) patients had diabetes during period of 10-19 years and the mean duration of diabetes was found $12.7 \pm 3.81$ years (Table II). 17(22.1\%) patients had Klebsiella pneumonia, 14(18.2\%) had Pseudomonas aeruginosa, 11(14.3\%) had Staphylococcus aureus, 10(13.0\%) had Escherichia coli, 6(7.8\%) had Coagulase-negative staphylococci and $8(10.4 \%)$ had Providencia spp. (Table III). In Klebsiella pneumonia organism $94.1 \%$ sensitivity to imipenem, $70.6 \%$ to amikacin, $70.6 \%$ to piperacillin-tazobactam, $64.7 \%$ to meropenem. In Pseudomonas aeruginosa organism $92.9 \%$ sensitivity to imipenem, $74.6 \%$ to piperacillintazobactam, $64.3 \%$ to amikacin, $57.1 \%$ to ciprofloxacin. In Staphylococcus aureus organism 63.6\% sensitivity to tetracycline, $54.5 \%$ to Ceftriaxone. In Escherichia coli $100 \%$ sensitivity to imipenem, $70 \%$ to amoxicillinclavulanic acid, amikacin, piperacillin-tazobactam. In Coagulase-negative Staphylococci $83.3 \%$ sensitivity to tetracycline, $66.7 \%$ to ceftriaxone. In Proteus mirabilis $100 \%$ sensitivity to tetracycline, amikacin, ceftriaxone, imipenem, piperacillin-tazobactam. In Enterococcus spp. $75.0 \%$ sensitivity to tetracycline. In Citrobacter spp. $100 \%$ sensitivity to imipenem (Table IV). 
Table I Demographic Profile, risk factors and clinical presentation of diabetic foot patients $(\mathrm{n}=77)$

\begin{tabular}{lcc}
\hline Age in years & Number & Percentage \\
\hline - $21-30$ yrs & 7 & 9.1 \\
- $\quad 31-40 \mathrm{yrs}$ & 8 & 10.4 \\
- $\quad 41-50 \mathrm{yrs}$ & 18 & 23.4 \\
- $\quad 51-60 \mathrm{yrs}$ & 27 & 35.1 \\
- $\quad 61-70 \mathrm{yrs}$ & 14 & 18.2 \\
- $\quad>70 \mathrm{yrs}$ & 3 & 3.9 \\
Mean $( \pm$ SD) & $51.57( \pm 12.13)$ & Range $21-78$ years \\
Sex & & \\
- $\quad$ Male & 54 & 70.1 \\
- Female & 23 & 29.9 \\
Risk factors & & \\
- Trauma & 50 & 64.9 \\
- Smoking & 23 & 29.9 \\
- Family history & 11 & \\
$\quad$ of diabetes & & \\
\hline
\end{tabular}

Table II Duration of diabetes mellitus ( $\mathrm{n}=77)$

Duration of diabetes Number Percentage (years) (Mean SD) 12.7( \pm 3.81$)$ Range 5-27 years

\begin{tabular}{lcc}
$<10 \mathrm{yrs}$ & 18 & 23.4 \\
$10-19 \mathrm{yrs}$ & 53 & 68.8 \\
$\geq 20 \mathrm{yrs}$ & 6 & 7.8 \\
\hline
\end{tabular}

Table III Bacteria isolated from diabetic foot ulcers

\begin{tabular}{lcc}
\hline & Number & Percentage \\
\hline Klebsiella pneumoniae & 17 & 22.1 \\
Pseudomonas aeruginosa & 14 & 18.2 \\
Staphylococcus aureus & 11 & 14.3 \\
Escherichia coli & 10 & 13.0 \\
Coagulase-negative staphylococci & 6 & 7.8 \\
Proteus mirabilis & 4 & 5.2 \\
Enterococcus spp. & 4 & 5.2 \\
Citrobacter spp. & 3 & 3.9 \\
Proteus vulgaris & 1 & 1.3 \\
Acinetobacter spp. & 1 & 1.3 \\
Pseudomonas spp. & 1 & 1.3 \\
Providencia spp. & 8 & 10.4 \\
\hline
\end{tabular}

Table IV Antimicrobial susceptibility pattern of aerobic bacterial isolates from infected foot in diabetic patients

Bacterial isolates Sensitivity pattern $(\%)$

AC TE CI TS GM AK NC CFX CTR CAZ IP PT COL FA LIN MER

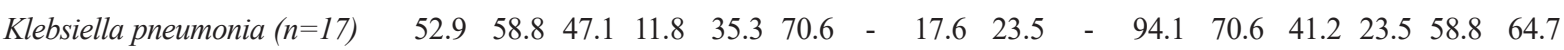

Pseudomonas aeruginosa $(n=14)$ - $\quad$ - $\quad 57.1 \quad$ - $\quad 50.064 .342 .9 \quad$ - $\quad$ - 28.692 .974 .650 .021 .442 .928 .6

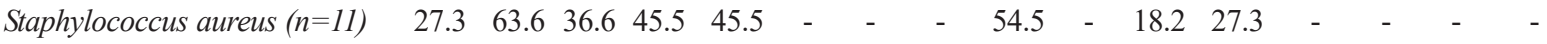

Escherichia coli $(n=-10)$

$\begin{array}{llllllllllllllll}70.0 & 30.0 & 30.0 & 20.0 & 40.0 & 70.0 & - & 20.0 & 30.0 & - & 100 & 70.0 & 30.0 & 40.0 & 20.0 & 50.0\end{array}$

Coagulase-negative

$\begin{array}{lllllllllllllllll}33.3 & 83.3 & 50.0 & 33.3 & 50.0 & - & - & - & 66.7 & - & 16.7 & - & - & - & 16.7 & 33.3\end{array}$

Staphylococci $(n=6)$

Proteus mirabilis $(n=4)$

$\begin{array}{llllllllllllllll}25.0 & 100 & 75.0 & 50.0 & 75.0 & 100 & - & 50.0 & 100 & - & 100 & 100 & 25.0 & - & 50.0 & 75.0\end{array}$

Enterococcus spp. $(n=4)$

$-75.050 .0-50.0$

Citrobacter spp. $(n=3)$

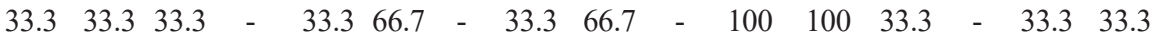

Proteus vulgaris $(n=1)$

$\begin{array}{lllllllllll}100 & 100 & 100 & - & - & - & - & -100 & - & 100\end{array}$

Acinetobacter spp. $(n=1)$

$-100-100-1 \quad-\quad-\quad-\quad-100100$

Pseudomonas spp. $(n=1)$

- $\quad$ - $100 \quad-\quad-\quad-100 \quad-\quad-\quad-100100$

Providencia spp. $(n=8)$

100 


\section{Discussion}

In this study majority $27(35.1 \%)$ patients belonged to age 51-60 years with mean age was found 51.57 \pm 12.13 years. Males were predominant $(70.1 \%)$, male: female ratio was 2.3:1. Almost two third (64.9\%) patients had trauma, 23(29.9\%) were smoker and 11(14.3\%) had family history of diabetes. Reghu et al. ${ }^{7}$ study observed that the age ranged from 25 to 93 years with mean age being 63.6 years. Males were predominant $(73.3 \%)$ in the study subjects. Umadevi et al. ${ }^{1}$ the age ranged from 32 to 73 years with mean age being $47 \pm 11$ years. Of the 105 patients with diabetic foot, $84(80 \%)$ were male and $21(20 \%)$ were female. Anand et al. ${ }^{2}$ study reported that the mean age of the patients was 52.42 years. The highest number of patients was in the older age group of 51-60 years old of 34\% (17/50). The male: female ratio is 3.5:1. Sixty-eight percent (34/50) of these patients had been diagnosed with diabetes for less than 10 years while the rest have been diagnosed for more than 10 years. This is in similar to the study conducted by Zaine et al. ${ }^{8}$ conducted in Sydney and Gardner et al. ${ }^{9}$ from Iowa where mean age of the study participants was 67 years and 64.2 years. In our study 23.4 percent $(18 / 77)$ of these patients had been diagnosed with diabetes for less than 10 years while the rest have been diagnosed for more than 10 years. Increasing age may be a contributory factor to chronic wounds as the skin can easily damage. Older cells do not proliferate as fast and may not have an adequate response to stress in terms of gene up regulation of stress related proteins. ${ }^{8-10}$ This is comparable to the study conducted by Perrin et al. ${ }^{11}$ from Victoria, where they reported male predominance at $61.3 \%$. The reason for this male predominance is unknown, although in Indian subcontinent, habit of bare foot walking and predominantly rural background may contribute to trauma leading to ulcers. Indians also sit with legs crossed for long hours of work or worship leading to repetitive, prolonged pressure over lateral malleolar areas, leading to bursae and dark hypertrophied skin, which can ulcerate and cause infection. ${ }^{11}$ Gadepalli et al. ${ }^{12}$ males were predominant $(85.0 \%)$ in the study subjects. The majority of subjects had type 2 diabetes $(88.8 \%)$. The mean age of the subjects was $53.9 \pm 12.1$ years. The mean duration of diabetes was $11.8 \pm 5.7$ years. In Bangladesh this male predominance is unknown, although in Bangladeshi peoples, habit of bare foot walking and predominantly rural background may contribute to trauma leading to ulcers. Bangladeshi also sit with legs crossed for long hours of work or worship leading to repetitive, prolonged pressure over lateral malleolar areas, leading to bursae and dark hypertrophied skin, which can ulcerate and cause infection.

In current study more than two third $(68.6 \%)$ patients had diabetes during period of 10-19 years and the mean duration of diabetes was found $12.7 \pm 3.81$ years. Gadepalli et al. ${ }^{12}$ the mean duration of diabetes was 11.8 \pm 5.7 years. Reghu et al. ${ }^{7}$ study observed that the duration of diabetes ranged from 1 year to 40 years with a mean duration of 16.2 years. Most of the patients (41.3\%) had a diabetic history of 11-20 years. Anand et al. ${ }^{2}$ reported that sixty-eight percent of these patients had been diagnosed with Diabetes for less than 10 years while the rest have been diagnosed for more than 10 years.

In current study showed $17(22.1 \%)$ patients had Klebsiella pneumonia, 14(18.2\%) had Pseudomonas aeruginosa, 11(14.3\%) had Staphylococcus aureus, 10(13.0\%) had Escherichia coli, 6(7.8\%) had Coagulase-negative staphylococci and $8(10.4 \%)$ had Providencia spp. In the Reghu et al. ${ }^{7}$ study, most of the isolated pathogens belonged to the genus Staphylococcus (20.1\%), Enterococcus (14.3\%) and Pseudomonas (13.6\%). The organisms isolated from infected diabetic foot ulcers. Among the Staphylococcus species, Staphylococcus aureus and Coagulase negative staphylococci constituted $9.2 \%$ and $7.0 \%$ of the isolates, respectively. Among Enterococcus and Pseudomonas species, Enterococcus faecalis and Pseudomonas aeruginosa constituted $12.1 \%$ and $11.4 \%$ of the isolates, respectively. Other commonly isolated organisms were Escherichia coli (12.1\%) and Klebsiella pneumonia $(10.2 \%)$. The Candida species isolated included Candida albicans (2.6\%), Candida parapsilosis and Candida tropicalis $(0.7 \%$ each); and Candida famata and Candida haemulonii (0.4\% each). Other organisms isolated included Proteus mirabilis (3.3\%), Acinetobacter baumannii (1.8\%), beta haemolytic streptococci (1.1\%) and Proteus vulgaris (0.7\%). Umadevi et al. ${ }^{1}$ study observed that Klebsiella pneumonia was found $20.5 \%$, Pseudomonas aeruginosa $17.0 \%$, Staphylococcus aureus $17.0 \%$, Escherichia coli $14.6 \%$, Coagulase-negative staphylococci $7.0 \%$, Proteus mirabilis 5.8\%, Enterococcus spp. 5.3\%, Citrobacter spp. 4.1, Proteus vulgaris 3.5\%, Acinetobacter spp. 
3.5\%, Pseudomonas spp. 1.2\%, Providencia spp. 0.6\%. In study of Anand et al. ${ }^{2}$ observed that on comparison of the bacterial isolate with the incident of amputation it was observed that $80 \%(4 / 5)$ of the patients in with Proteus mirabilis was isolated underwent amputation, $57.12 \%$ (4/7) with Streptococcus pyogenes, 50\% (1/2) with Pseudomonas aeruginosa, 33.3\% (2/6) with Klebsiella pneumonia, 12\% (2/16) with Staphylococcus aureus underwent amputation.

In this study observed that Klebsiella pneumonia organism $94.1 \%$ sensitivity to imipenem, $70.6 \%$ to amikacin, $70.6 \%$ to piperacillin-tazobactam, $64.7 \%$ to meropenem. In Pseudomonas aeruginosa organism $92.9 \%$ sensitivity to imipenem, $74.6 \%$ to piperacillintazobactam, $64.3 \%$ to amikacin, $57.1 \%$ to ciprofloxacin. In Staphylococcus aureus organism $63.6 \%$ sensitivity to tetracycline, $54.5 \%$ to ceftriaxone. In Escherichia coli $100 \%$ sensitivity to imipenem, $70 \%$ to amoxicillinclavulanic acid, amikacin, piperacillin-tazobactam. In Coagulase-negative Staphylococci $83.3 \%$ sensitivity to tetracycline, $66.7 \%$ to ceftriaxone. In Proteus mirabilis $100 \%$ sensitivity to tetracycline, amikacin, ceftriaxone, imipenem, piperacillin-tazobactam. In Enterococcus spp. $75.0 \%$ sensitivity to tetracycline. In Citrobacter spp. $100 \%$ sensitivity to imipenem. Reghu et al. ${ }^{7}$ study reported that the antibiotic susceptibility pattern of the isolates is also essential for proper management of diabetic foot infections. Against gram positive organisms linezolid, teicoplanin, tigecycline and vancomycin showed $>90 \%$ susceptibility. In their study, all Staphylococcus species isolated were susceptible to vancomycin, tigecycline, teicoplanin and linezolid and all of Enterococcus species susceptible to vancomycin. These antibiotics are highly effective against gram positive organisms isolated from this study and these antibiotics seem to be appropriate for empirical treatment of diabetic foot infections. Coagulase negative staphylococcus also showed $100 \%$ susceptibility to levofloxacin. Most of the gram positive organisms showed low susceptibility to erythromycin and Penicillin G. In the Reghu et al. ${ }^{7}$ study, against Pseudomonas species, colistin and amikacin showed good susceptibility. However, against Klebsiella species, amikacin showed only 58\% susceptibility. Klebsiella, Escherichia coli and Acinetobacter isolates were susceptible to colistin. Majority of the Klebsiella and Acinetobacter isolates were resistant to cefoperazone sulbactam, co-trimoxazole, and piperacillin tazobactam. Against Escherichia coli, meropenem and amikacin showed $>80 \%$ susceptibility. Proteus species showed $100 \%$ susceptibility to amikacin and levofloxacin. Acinetobacter species showed complete resistance to levofloxacin. Management of gram negative infections is extremely challenging. Future studies should aim at identifying the risk factors for the development of these infections, so that appropriate treatment can be implemented early and can hence prevent fatal outcomes. The antibiotic susceptibility pattern of the gram negative bacteria isolated from diabetic ulcers. Against Candida species, amphotericin and fluconazole showed $83.3 \%$ and $90.9 \%$ susceptibility, respectively. Umadevi et al. ${ }^{1}$ majority of isolates of Escherichia coli and Klebsiella pneumoniae were susceptible to amikacin, piperacillin-tazobactam and imipenem, but resistant to other antibiotics tested except amoxicillinclavulanic acid for which they were showing variable susceptibility. Similarly, most of our Proteus spp. were susceptible to tetracycline, ciprofloxacin, amikacin, ceftriaxone, piperacillin-tazobactam and imipenem, while being less susceptible to amoxicillin-clavulanic acid, trimethoprim-sulfamethoxazole and cefuroxime. However, Proteus mirabilis was relatively more susceptible than Proteus vulgaris to most antibiotics. Citrobacter spp. were susceptible to piperacillintazobactam, amikacin, ceftriaxone and imipenem, but resistant to other antibiotics tested.

Most of the Pseudomonas aeruginosa were susceptible to piperacillin-tazobactam and imipenem, while they were showing varying susceptibility to ciprofloxacin, gentamicin, amikacin and netilmicin. Similarly, majority of Acinetobacter spp. were susceptible to piperacillintazobactam, imipenem and trimethoprimsulfamethoxazole, while being less susceptible to gentamicin, amikacin, ciprofloxacin, tetracycline, ceftiaxone and ceftazidime. The antibiotic susceptibility patterns of the grampositive bacteria isolated from diabetic ulcers. Staphylococcus aureus were most often susceptible to erythromycin, tetracycline and vancomycin, but were relatively less susceptible to amoxicillin-clavulanic acid, trimethoprimsulfamethoxazole, ciprofloxacin, gentamicin and ceftriaxone. None of the Staphylococcus aureus were susceptible to penicillin. Most of the Enterococcus spp. were susceptible only to vancomycin. However they 
showed varying susceptibility to tetracycline, penicillin, and ciprofloxacin. High-level aminoglycoside resistance was observed in $33 \%$ of the Enterococcus spp. ${ }^{1}$ Yerat and Rangasamy ${ }^{5}$ study observed that regarding the antimicrobial sensitivity pattern, we found that $57.14 \%$ of $S$. aureus were beta lactamase producer and 5 of the 21 isolates were methicillin-resistant $S$. aureus (MRSA) (23.80\%). The GPC isolates were $100 \%$ sensitive to vancomycin while all the Gram-negative bacterial isolates were $100 \%$ sensitive to imipenem.

\section{Conclusion}

Common organism found in diabetic foot ulcer patients were Klebsiella pneumonia, Pseudomonas aeruginosa, Staphylococcus aureus, Escherichia coli, Coagulasenegative staphylococci and Providencia spp. In tetracycline, amikacin, ceftriaxone, imipenem, piperacillin-tazobactam were $100 \%$ sensitive in Proteus mirabilis and only imipenem found in Escherichia coli and Citrobacter spp.

Conflict of interest: Nothing to declare.

\section{References}

1. Umadevi S, Kumar S, Joseph NM, Easow JM, Kandhakumari G, Srirangaraj S, Raj S, Stephen S. Microbiological study of diabetic foot infections. Indian Journal of Medical Specialities 2011; 2:12-17.

2. Anand A, Biswal I, Soni RK, Sinha A, Rynga D, Deb M. A clinico-microbiological study of diabetic foot ulcer patients to identify risk factors and their correlation with prognosis in tertiary care hospital in India. Int Surg J. 2016; 3:669-673.
3. Aherrao N, Shahi SK, Dwivedi A, Kumar A, Gupta S, Singh SK. Detection of anaerobic infection in diabetic foot ulcer using PCR technique and the status of metronidazole therapy on treatment outcome. Wounds 2012; 24 : 283-8.

4. Haldar J, Mukherjee P, Mukhopadhyay S, Maiti PK. Isolation of bacteria from diabetic foot ulcers with special reference to anaerobe isolation by simple two-step combustion technique in candle jar. Indian J Med Res 2017; 145:97-101.

5. Yerat RC, Rangasamy VR. A clinicomicrobial study of diabetic foot ulcer infections in South India. Int J Med Public Health 2015; 5:236-41.

6. Lipsky BA, Berendt AR, Cornia PB, Pile JC, Peters EJ, Armstrong DG, et al. 2012 Infectious Diseases Society of America clinical practice guideline for the diagnosis and treatment of diabetic foot infections. Clin Infect Dis 2012; 54:e132-73.

7. Reghu R, Padma UD, Sasankan V, Puthur S, Jose J. A Microbiological Study of Diabetic Foot Ulcer in a South Indian Tertiary Care Hospital. Int. J. Pharm. Sci. Rev. Res., 2016; 37: 167-170.

8. Zaine NH, Burns J, Vicaretti M, Fletcher JP, Begg L, Hitos K. Characteristics of diabetic foot ulcers in Western Sydney, Australia. Journal of Foot and Ankle Research. 2014; 7:39.

9. Gardner SE, Hillis SL, Heilmann K, Segre JA, Grice EA. The neuropathic diabetic foot ulcer micro biome is associated with clinical factors. Diabetes. 2013; 62:923-30.

10. Posnett J, Franks PJ. The burden of chronic wounds in the UK. Nurs Times. 2008; 104:44-5.

11. Perrin B. A retrospective audit of a diabetic foot clinic. Australas J Podiatr Med. 2006;40:23-5.

12. Gadepalli R, Dhawan B, Sreenivas V, Kapil A, Ammini AC, Chaudhry R. A Clinico-microbiological Study of Diabetic Foot Ulcers in an Indian Tertiary Care Hospital. Diabetes Care 2006; 29:1727-32. 